
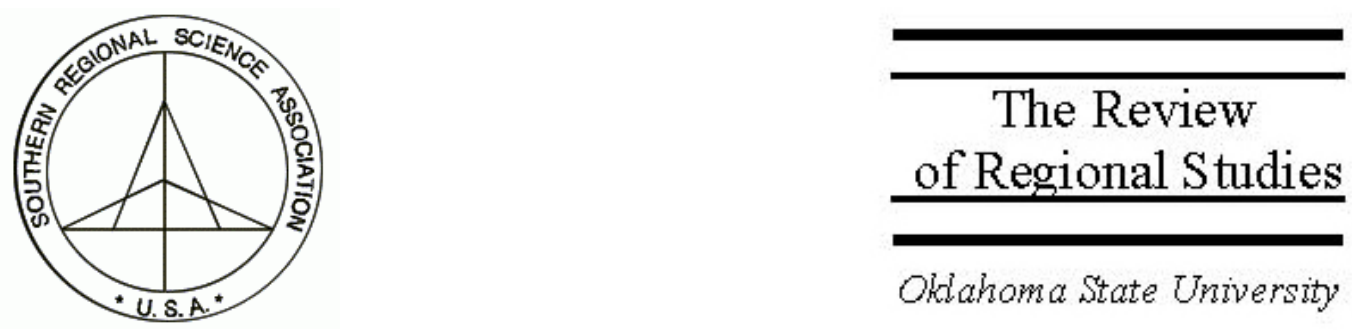

\title{
Frontier Issues in Rural and Regional Development
}

After several years of organizing the Rural Development and Population sessions at the North American Regional Science Association conferences, we were approached by the editors of the Review to construct a special issue from the 2006 sessions held in Toronto, Canada. We welcomed this much-appreciated opportunity to showcase the frontier research that comprises the grist of these engaging sessions, as scholars present their freshest ideas to regional science's preeminent international audience every year. We chose the top papers from these sessions, conducted double-blind reviews with a set of outstanding referees, and selected the final papers for invitations for revision and resubmission. We now take advantage of the Review's online format to get these peerreviewed pieces of cutting-edge research to this journal's readership while still literally warm from their respective cognitive ovens.

We are especially delighted to be able to share this research with the Review's readers given their natural interest and contributions to the rural development field. The Southern Regional Science Association remains the group perhaps most associated with significant advances in rural scholarship, so it is fitting to be able to highlight this field's most recent ground-breaking work in the pages of the association's flagship journal. Furthermore, the Review has done a tremendous service in keeping the regional science community and affiliated fields apprised of just how innovative and interesting rural topics can be, both in themselves and their lessons for broader regional and urban analysis.

The five papers in this issue span the range of such innovations and interests, in topics as well as methodology. The first paper on "Regional Unemployment Clusters: Neighbourhood and State Effects in Europe and North America," by Jose Enrique Garcilazo and Vincenzo Spiezia, applies a revealing nonparametric analysis to better understand the long-sought sources of differing regional distributions in unemployment 
rates. Recent work suggests that the regional distribution of unemployment rates is more dependent on geographic location (neighborhood effects) than on national factors (state factors). However, given the likelihood that state and neighborhood effects are not independent, this paper's innovative approach constructs a probability estimate of their joint effects to help isolate each factor's role. Using this estimate, they construct a simple measure that is invariant in space and across time to compare state, neighborhood, and joint effects in the determination of Europe and North America unemployment rates. The key finding is that neighborhood effects are stronger than state effects in Europe, while the interdependent joint effects dominate both polar characteristics in North America.

The second paper, by Tomas Jensen and Steven Deller, tackles "Spatial Modeling of the Migration of Older Persons with a Focus on Amenities." As in the previous paper, innovative methods (in this case, Geographically Weighted Regressions, or GWRs) meet a front-burner demographic and policy topic, namely the migration of a gathering babyboomer wave of older Americans. They find significant spatial variation in the amenity drivers of older person migration, leveraging the GWRs' flexibility in allowing sensitivity of responses of particular drivers to vary regionally. In particular, older persons seem to migrate towards combinations of built amenities in combination with natural amenities, in contrast to other studies of broader age profiles that have tended to only find consistent evidence of responses to natural features.

The third paper, by Vincenzo Spiezia and Stephan Weiler, tries to provide additional "Understanding [of] Regional Growth." Again, the combination of methodology, a hybrid growth-decomposition approach similar to shift-share, is coupled with an ongoing core regional analysis question, namely identifying the sources of economic growth. The hybrid methodology, now regularly used by OECD analysts, is developed in the first part of the paper, and then applied to OECD subnational regions to assess the relative importance of various growth factors. The most revealing results stem from the analysis of the cross-national sources of growth in the fastest and slowest growing regions over 19982003, with particular attention to the method's useful isolation of the region-specific assets that are most influenced by policy. Based on these findings, the paper concludes with challenges to several "conventional wisdoms" regarding sources of regional growth.

The fourth paper, by Anderson, Weng, and Goe, develops key steps towards a mixed quantitative/qualitative approach to analyze the context of the working poor. "Multidimensional Research Strategies for Understanding the Changing Rates of the Working Poor across the North Central United States” sets the stage by conducting an econometric analysis of the evolution of the rate of the working poor across the 1055 counties of the region between 1990 and 2000. Using the regression model as a benchmark, the authors highlight those outlier counties that had unusually strong increases or decreases in those rates that could not be explained by the spatial, labor market, or demographic characteristics of the region.

Loveridge et al. then consider these same regions facing "Advances and Declines in the Working Poor" through intensive case studies of a subsample of the noted outlier 
counties to complement Anderson, Weng, and Goe's quantitative work. The value-added of this ground-level qualitative inquiry underscores the analytical value of meshing traditional cross-sectional econometric techniques with more in-depth case study analyses. Loveridge et al. find that self-reliance, regional industrial history, and the relative prospects of neighboring regions are among the distinct factors that may be driving the changing rates of working poor in the outlier counties.

In sum, each of these five papers meshes a methodological innovation with a topic of ongoing policy and/or analytical interest, highlighting the fact that rural development research remains fresh in terms of both approach and application. In that sense, the growth and stagnation issues facing rural regions may in fact help inform broader regional and urban policy debates through both their similarities and differences in contexts, pasts, and prospects. Furthermore, the fact that successful rural areas tend to "graduate" to metropolitan status makes understanding the difficulties of the remaining rural areas that much more important as scholars and analysts seek to discover why some regions succeed while others continue to struggle.

Peter Schaeffer

Stephan Weiler 